\title{
Existence and multiple solutions to a discrete fourth order boundary value problem
}

\author{
Xia Liu' ${ }^{12^{*}}$, Tao Zhou ${ }^{3}$ and Haiping Shi ${ }^{4,5}$
}

\section{"Correspondence:}

xia991002@163.com

${ }^{1}$ College of Continuing Education and Open College, Guangdong

University of Foreign Studies,

Guangzhou, China

${ }^{2}$ Science College, Hunan

Agricultural University, Changsha,

China

Full list of author information is

available at the end of the article

\begin{abstract}
In this article, we study a discrete fourth order boundary value problem. By making use of variational methods and critical point theory, we obtain some criteria for the existence and multiple solutions. Moreover, two examples are included to illustrate the applicability of the main results.
\end{abstract}

MSC: 39A10; 34B05; 58E05; 65L10

Keywords: Boundary value problems; Existence and multiple solutions; Fourth order; Critical point theory; Discrete

\section{Introduction and statement of the main results}

In this article, we are interested in the existence and multiple solutions to the discrete fourth order nonlinear equation

$$
\Delta^{4} u_{n-2}-\Delta\left(r_{n-1} \Delta u_{n-1}\right)=f\left(n, u_{n}\right), \quad n \in \mathbb{Z}[1, k]
$$

with boundary value conditions

$$
\Delta^{i} u_{-1}=\Delta^{i} u_{k-1}, \quad i=0,1,2,3,
$$

where $\Delta^{j} u_{n}=\Delta\left(\Delta^{j-1} u_{n}\right)(j=2,3,4), \Delta^{0} u_{n}=u_{n}, \Delta u_{n}=u_{n+1}-u_{n}, f(s, u) \in C\left(\mathbb{R}^{2}, \mathbb{R}\right), r_{n}>0$ is real-valued for each $n \in \mathbb{Z}[0, k], r_{0}=r_{k}, k \geq 1$ is an integer. Here, $\mathbb{Z}$ denotes the sets of integers, $\mathbb{R}$ denotes the sets of real numbers, $\mathbb{N}$ denotes the sets of natural numbers. Given $a \leq b$ in $\mathbb{Z}$, let $\mathbb{Z}[a, b]:=\mathbb{Z} \cap[a, b]$. Let $u^{*}$ denote the transpose of a vector $u$.

Boundary value problem (1.1) with (1.2) can be regarded as being a discrete analogue of the fourth order differential equation

$$
u^{(4)}(s)-\left[r(s) u^{\prime}(s)\right]^{\prime}=f(s, u(s)), \quad s \in(0,1),
$$

with boundary value conditions

$$
u^{(i)}(0)=u^{(i)}(1), \quad i=0,1,2,3
$$

(c) The Author(s) 2018. This article is distributed under the terms of the Creative Commons Attribution 4.0 International License (http://creativecommons.org/licenses/by/4.0/), which permits unrestricted use, distribution, and reproduction in any medium, provided you give appropriate credit to the original author(s) and the source, provide a link to the Creative Commons license, and indicate if changes were made. 
(1.3) includes the following equation:

$$
u^{(4)}(s)=f(s, u(s)), \quad s \in \mathbb{R},
$$

which is used to describe the stationary states of the deflection of an elastic beam [29]. Differential equations similar to (1.3) and special cases of it have been studied using a number of different methods in the literature, we refer the reader to papers $[1,2,11-14$, $24,25]$ and the references contained therein.

Difference equations $[1-10,15-20,22,26-28,30,31]$ appear in numerous settings and forms, both as a fundamental tool in the discrete analogue of a differential equation and as a useful model for several economical and population problems.

If $f\left(n, u_{n}\right)=q_{n} u_{n}$, Peterson and Ridenhour [22] considered the fourth order difference equation

$$
\Delta^{4} u_{n-2}+q_{n} u_{n}=0, \quad n \in[a+2, a+3, \ldots, b+2],
$$

and gave some conditions on $q_{n}$ that ensure (1.6) is (2,2)-disconjugate on $[a, b+4]$ utilizing an appropriately defined quadratic form.

Making use of the symmetric mountain pass lemma, Chen and Tang [5] established some existence criteria to guarantee the fourth order difference system

$$
\Delta^{4} u_{n-2}+q_{n} u_{n}=f\left(n, u_{n+1}, u_{n}, u_{n-1}\right), \quad n \in \mathbb{Z}
$$

has infinitely many homoclinic orbits.

In [16], the existence, multiplicity, and nonexistence results of nontrivial solutions for discrete nonlinear fourth order boundary value problems

$$
\Delta^{4} u_{n-2}+\eta \Delta^{2} u_{n-1}-\xi u_{n}=\lambda f\left(n, u_{n}\right), \quad n \in \mathbb{Z}[a+1, b+1],
$$

with

$$
u_{a}=\Delta^{2} u_{a-1}=0, \quad u_{b+2}=\Delta^{2} u_{b+1}=0,
$$

are obtained. The methods used here are based on the critical point theory and monotone operator theory.

Positive solutions of the following fourth order nonlinear difference equations with a deviating argument

$$
\Delta a_{n}\left(\Delta b_{n}\left(\Delta c_{n}\left(\Delta u_{n}\right)^{\gamma}\right)^{\beta}\right)^{\alpha}+d_{n} u_{n+\tau}^{\lambda}=0
$$

are investigated. Došlá, Krejčová, and Marini [8] introduced for (1.8) the notions of a minimal solution and a maximal solution, and gave necessary and sufficient conditions for their existence. Some relationships with nonoscillatory solutions, which have a different growth at infinity, were presented as well. 
Graef, Kong, and Wang [10] studied the discrete fourth order periodic boundary value problem with a parameter

$$
\left\{\begin{array}{l}
\Delta^{4} u_{n-2}-\Delta\left(p_{n-1} \Delta u_{n-1}\right)+q_{n} u_{n}=\lambda f\left(n, u_{n}\right), \quad n \in \mathbb{Z}(1, N) \\
\Delta^{i} u_{-1}=\Delta^{i} u_{N-1}, \quad i=0,1,2,3
\end{array}\right.
$$

By using variational methods and the mountain pass lemma, sufficient conditions are found under which the above problem has at least two nontrivial solutions.

In 2015, Liu, Zhang, and Shi [19] considered the following fourth order nonlinear difference equation:

$$
\Delta^{2}\left(p_{n-2} \Delta^{2} u_{n-2}\right)-\Delta\left(q_{n-1} \Delta u_{n-1}\right)=f\left(n, u_{n+1}, u_{n}, u_{n-1}\right), \quad n \in \mathbb{Z}(1, k),
$$

with boundary value conditions

$$
u_{-1}=u_{0}=0, \quad u_{k+1}=u_{k+2}=0 .
$$

Using the critical point theory, the authors established various sets of sufficient conditions for the existence and nonexistence of solutions for the Dirichlet boundary value problem and gave some new results.

By using the invariant set of descending flow and variational method, Long and Chen [20] in 2018 established the existence of multiple solutions to a class of second order discrete Neumann boundary value problem

$$
\left\{\begin{array}{l}
-\Delta\left(p_{n-1} \Delta u_{n-1}\right)+q_{n} u_{n}=k f\left(n, u_{n}\right), \quad n \in \mathbb{Z}(1, N), \\
\Delta u_{0}=\Delta u_{N} .
\end{array}\right.
$$

The solutions included sign-changing solutions, positive solutions, and negative solutions. Moreover, an example was given to illustrate our results.

In the last few years, variational methods and critical point theory have been used to study the existence and multiple solutions of discrete boundary value problems. In this article, we utilize this approach to obtain some sufficient conditions for the existence and multiple solutions to the boundary value problem (BVP for short) (1.1) with (1.2). What is more, two examples are included to illustrate the applicability of the main results.

Throughout this article, assume that there is a function $F(s, u)$ such that

$$
F(s, u)=\int_{0}^{u} f(s, t) d t
$$

for any $(s, u) \in \mathbb{R}^{2}$.

Our main results are the following theorems.

Theorem 1.1 Assume that the function $F(s, u) \geq 0$ satisfies the following assumptions:

$\left(F_{1}\right)$ There exist two constants $\delta_{1}>0$ and $a_{1} \in\left(0, \frac{\lambda_{\min }}{2}\right)$ such that

$$
F(s, u) \leq a_{1} u^{2}, \quad \forall s \in \mathbb{R}^{2},|u| \leq \delta_{1}
$$


$\left(F_{2}\right)$ There exist two constants $a_{2} \in\left(\frac{\lambda_{\max }}{2},+\infty\right)$ and $a_{3}>0$ such that

$$
F(s, u) \geq a_{2} u^{2}-a_{3}, \quad \forall(s, u) \in \mathbb{R}^{2}
$$

where $\lambda_{\min }$ and $\lambda_{\max }$ are constants which can be referred to (2.4) and (2.5).

Then BVP (1.1) with (1.2) admits at least three solutions which are a trivial solution and two nontrivial solutions.

Remark 1.1 In [10], the authors considered the discrete fourth order periodic boundary value problem with a parameter

$$
\left\{\begin{array}{l}
\Delta^{4} u_{n-2}-\Delta\left(p_{n-1} \Delta u_{n-1}\right)+q_{n} u_{n}=\lambda f\left(n, u_{n}\right), \quad n \in \mathbb{Z}(1, N), \\
\Delta^{i} u_{-1}=\Delta^{i} u_{N-1}, \quad i=0,1,2,3 .
\end{array}\right.
$$

The following hypotheses are satisfied in [10]:

(H1) $p_{n}>0$ for $n \in \mathbb{Z}(0, N+1)$ and $q_{n}>0$ for $n \in \mathbb{Z}(0, N)$;

(H2) $\lim _{|n| \rightarrow \infty} \frac{|F(n, u)|}{|u|^{2}}=0$ for $n \in \mathbb{Z}(0, N)$;

(H3) $\lim \sup _{|n| \rightarrow \infty} \frac{|F(n, u)|}{|u|^{2}} \leq 0$ for $n \in \mathbb{Z}(0, N)$;

(H4) there exists $\omega \in U$ such that $\sum_{n=1}^{N} F\left(n, \omega_{n}\right)>0$.

Note that $\left(F_{2}\right)$ of Theorem 1.1 does not satisfy $(H 2)$. At least two nontrivial solutions of (1.9) are obtained by the mountain pass lemma in [10]. However, in our paper, we employ a linking theorem to obtain at least two nontrivial solutions. Furthermore, our conditions on the nonlinear term are weaker than [10].

Theorem 1.2 Assume that the function $F(s, u) \geq 0$ satisfies the following assumptions:

$\left(F_{3}\right) \lim _{|u| \rightarrow 0} \frac{F(s, u)}{u^{2}}=0, \forall(s, u) \in \mathbb{R}^{2}$.

$\left(F_{4}\right)$ There exist three constants $a_{4}>0, \gamma>2$, and $a_{5}>0$ such that

$$
F(s, u) \geq a_{4}|u|^{\gamma}-a_{5}, \quad \forall(s, u) \in \mathbb{R}^{2}
$$

Then BVP (1.1) with (1.2) admits at least three solutions which are a trivial solution and two nontrivial solutions.

Theorem 1.3 Assume that the function $F(s, u) \geq 0,\left(F_{1}\right)$ and $\left(F_{2}\right)$ and the following assumptions are satisfied:

(f) $f(s,-u)=-f(s, u), \quad \forall(s, u) \in \mathbb{R}^{2}$.

Then BVP (1.1) with (1.2) admits at least q distinct pairs of nontrivial solutions, where $q$ is the dimension of $Q$ which can be referred to (2.6).

Remark 1.2 In [9], the authors considered the fourth order nonlinear difference equation

$$
\left\{\begin{array}{l}
\Delta^{4} u_{n-2}-\Delta\left(p_{n-1} \Delta u_{n-1}\right)+q_{n} u_{n}=f\left(n, u_{n}\right), \quad n \in \mathbb{Z}(1, N) \\
\Delta^{i} u_{-1}=\Delta^{i} u_{N-1}, \quad i=0,1,2,3
\end{array}\right.
$$


Define $f_{0}=\liminf _{u \rightarrow 0} \min _{n \in \mathbb{Z}(1, N)} \frac{f(n, u)}{u}$ and $f^{\infty}=\lim \sup _{u \rightarrow 0} \max _{n \in \mathbb{Z}(1, N)} \frac{f(n, u)}{u}$.

The following hypotheses are satisfied in [9]:

$\left(H_{1}\right) p_{n} \geq 0$ and $q_{n} \geq 0$ for $n \in \mathbb{Z}(1, N)$ and there exists $\eta$ with $\eta<\underline{q}$ such that $f^{\infty} \leq \eta$, where $\underline{q}=\min _{n \in \mathbb{Z}(1, N)} q_{n}$;

$\left(H_{2}\right) f(n, u)$ is odd in $u$, i.e., $f(n,-u)=-f(n, u)$ for $(n, u) \in \mathbb{Z}(1, N) \times \mathbb{R}$;

$\left(H_{3}\right)$ there exists $m \in\{1, \ldots, N\}$ such that $f_{0}>\lambda_{m}$.

Note that $\left(F_{1}\right)$ of Theorem 1.3 does not satisfy $\left(H_{3}\right)$. Furthermore, our conditions on the nonlinear term are weaker than [9].

If $f\left(n, u_{n}\right)=\tau_{n} \psi\left(u_{n}\right),(1.1)$ reduces to the following fourth order nonlinear equation:

$$
\Delta^{4} u_{n-2}-\Delta\left(r_{n-1} \Delta u_{n-1}\right)=\tau_{n} \psi\left(u_{n}\right), \quad n \in \mathbb{Z}[1, k],
$$

where $\psi \in C(\mathbb{R}, \mathbb{R}), \tau_{n}>0$ is real-valued for each $n \in \mathbb{Z}[1, k]$. Therefore, we can easily obtain the following results.

Theorem 1.4 Assume that the following assumptions are satisfied:

$\left(\Psi_{1}\right)$ There exists a function $\Psi(u) \in C^{1}(\mathbb{R}, \mathbb{R})$ with $\Psi(u) \geq 0$ such that

$$
\Psi^{\prime}(u)=\psi(u)
$$

$\left(\Psi_{2}\right)$ There exist two constants $\delta_{2}>0$ and $a_{6} \in\left(0, \frac{\lambda_{\min }}{2}\right)$ such that

$$
\Psi(u) \leq a_{6} u^{2}, \quad \forall s \in \mathbb{R}^{2},|u| \leq \delta_{2} .
$$

$\left(\Psi_{3}\right)$ There exist two constants $a_{7} \in\left(\frac{\lambda_{\max }}{2},+\infty\right)$ and $a_{8}>0$ such that

$$
\Psi(u) \geq a_{7} u^{2}-a_{8}, \quad \forall(s, u) \in \mathbb{R}^{2},
$$

where $\lambda_{\min }$ and $\lambda_{\max }$ are constants which can be referred to (2.4) and (2.5).

Then BVP (1.10) with (1.2) admits at least three solutions which are a trivial solution and two nontrivial solutions.

Corollary 1.1 Assume that $\left(\Psi_{1}\right),\left(\Psi_{2}\right),\left(\Psi_{3}\right)$ and the following assumption are satisfied.

$(\psi) \quad \psi(-u)=-\psi(u), \quad \forall u \in \mathbb{R}$.

Then BVP (1.10) with (1.2) admits at least $q$ distinct pairs of nontrivial solutions, where $q$ is the dimension of $Q$ which can be referred to (2.6).

\section{Variational framework}

In this section, we shall establish the corresponding variational framework for BVP (1.1) with (1.2) which will be of fundamental importance in proving our main results.

In order to apply the critical point theory, we define a $k$-dimensional Hilbert space $U$ by

$$
U:=\left\{u: \mathbb{Z}[-1, k+2] \rightarrow \mathbb{R} \mid \Delta^{i} u_{-1}=\Delta^{i} u_{k-1}, i=0,1,2,3\right\},
$$


and equip it with the inner product

$$
(u, v):=\sum_{j=1}^{k} u_{j} v_{j}, \quad \forall u, v \in U
$$

and the induced norm

$$
\|u\|:=\left(\sum_{j=1}^{k} u_{j}^{2}\right)^{\frac{1}{2}}, \quad \forall u \in U .
$$

Remark 2.1 It is obvious that

$$
u_{-1}=u_{k-1}, \quad u_{0}=u_{k}, \quad u_{1}=u_{k+1}, \quad u_{2}=u_{k+2}, \quad \forall u \in U \text {. }
$$

As a matter of fact, $U$ is isomorphic to $\mathbb{R}^{k}$. Throughout this article, when we say $u=$ $\left(u_{1}, u_{2}, \ldots, u_{k}\right) \in \mathbb{R}^{k}$, we always imply that $u$ can be extended to a vector in $U$ so that (2.1) holds.

Define a functional $J$ on $U$ by

$$
J(u):=\frac{1}{2} \sum_{n=1}^{k}\left(\Delta^{2} u_{n-2}\right)^{2}+\sum_{n=1}^{k} r_{n-1}\left(\Delta u_{n-1}\right)^{2}-\sum_{n=1}^{k} F\left(n, u_{n}\right)
$$

After a careful computation, we have

$$
\frac{\partial J}{\partial u_{n}}=\Delta^{4} u_{n-2}-\Delta\left(r_{n-1} \Delta u_{n-1}\right)-f\left(n, u_{n}\right), \quad n \in \mathbb{Z}[1, k] .
$$

Therefore, $J^{\prime}(u)=0$ if and only if

$$
\Delta^{4} u_{n-2}-\Delta\left(r_{n-1} \Delta u_{n-1}\right)=f\left(n, u_{n}\right), \quad n \in \mathbb{Z}[1, k]
$$

Consequently, we reduce the problem of finding a solution of BVP (1.1) with (1.2) to that of seeking a critical point of the functional $J$ on $U$. Denote the $k \times k$ matrices $S$ and $R$.

For $k=1$, let $S=R=(0)$.

For $k=2$, let

$$
S=\left(\begin{array}{cc}
8 & -8 \\
-8 & 8
\end{array}\right)
$$

and

$$
R=\left(\begin{array}{cc}
r_{0}+r_{1} & -r_{0}-r_{1} \\
-r_{0}-r_{1} & r_{0}+r_{1}
\end{array}\right)
$$

For $k=3$, let

$$
S=\left(\begin{array}{ccc}
6 & -3 & -3 \\
-3 & 6 & -3 \\
-3 & -3 & 6
\end{array}\right)
$$


For $k=4$, let

$$
S=\left(\begin{array}{cccc}
6 & -4 & 2 & -4 \\
-4 & 6 & -4 & 2 \\
2 & -4 & 6 & -4 \\
-4 & 2 & -4 & 6
\end{array}\right)
$$

For $k \geq 5$, let

$$
S=\left(\begin{array}{cccccccccc}
6 & -4 & 1 & 0 & 0 & \ldots & 0 & 0 & 1 & -4 \\
-4 & 6 & -4 & 1 & 0 & \ldots & 0 & 0 & 0 & 1 \\
1 & -4 & 6 & -4 & 1 & \ldots & 0 & 0 & 0 & 0 \\
0 & 1 & -4 & 6 & -4 & \ldots & 0 & 0 & 0 & 0 \\
0 & 0 & 1 & -4 & 6 & \ldots & 0 & 0 & 0 & 0 \\
\ldots & \ldots & \ldots & \ldots & \ldots & \ldots & \ldots & \ldots & \ldots & \ldots \\
0 & 0 & 0 & 0 & 0 & \ldots & 6 & -4 & 1 & 0 \\
0 & 0 & 0 & 0 & 0 & \ldots & -4 & 6 & -4 & 1 \\
1 & 0 & 0 & 0 & 0 & \ldots & 1 & -4 & 6 & -4 \\
-4 & 1 & 0 & 0 & 0 & \ldots & 0 & 1 & -4 & 6
\end{array}\right) .
$$

For $k \geq 3$, let

$$
R=\left(\begin{array}{ccccc}
r_{0}+r_{1} & -r_{1} & 0 & \cdots & -r_{0} \\
-r_{1} & r_{1}+r_{2} & -r_{2} & \ldots & 0 \\
0 & -r_{2} & r_{2}+r_{3} & \ldots & 0 \\
\cdots & \ldots & \ldots & \ldots & \ldots \\
0 & 0 & 0 & \ldots & -r_{k-1} \\
-r_{0} & 0 & 0 & \ldots & r_{k-1}+r_{0}
\end{array}\right)
$$

Let $M:=S+R$. We rewrite $J(u)$ as

$$
J(u)=\frac{1}{2} u^{*} M u-\sum_{n=1}^{k} F\left(n, u_{n}\right) .
$$

It is easy to see that 0 is an eigenvalue of $M,(1,1, \ldots, 1)^{*}$ is an eigenvector associated with $0 . M$ is semi-positive definite. Let $\lambda_{1}, \lambda_{2}, \ldots, \lambda_{k}$ be the eigenvalues of $M$.

Set

$$
\lambda_{\min }=\min \left\{\lambda_{j} \mid \lambda_{j} \neq 0, j=1,2, \ldots, k\right\},
$$

and

$$
\lambda_{\max }=\max \left\{\lambda_{j} \mid \lambda_{j} \neq 0, j=1,2, \ldots, k\right\} .
$$

Let $P=\left\{(c, c, \ldots, c)^{*} \in U \mid c \in \mathbb{R}\right\}$, then $P$ is an invariant subspace of $U$. Denote $Q$ by

$$
U=P \oplus Q
$$




\section{Some basic lemmas}

Assume that $U$ is a real Banach space and $J \in C^{1}(U, \mathbb{R})$. As usual, $J$ is said to satisfy the Palais-Smale condition if every sequence $\left\{u^{(j)}\right\} \subset U$ such that $\left\{J\left(u^{(j)}\right)\right\}$ is bounded and $J^{\prime}\left(u^{(j)}\right) \rightarrow 0(j \rightarrow \infty)$ has a convergent subsequence. The sequence $\left\{u^{(j)}\right\}$ is called a PalaisSmale sequence.

Let $U$ be a real Banach space. Define the symbol $B_{\rho}(u)$ as the open ball in $U$ about $u$ of radius $\rho, \partial B_{\rho}(u)$ as its boundary, and $\bar{B}_{\rho}(u)$ as its closure.

Lemma 3.1 (Linking theorem $[21,23])$ Let $U$ be a real Banach space, $U=U_{1} \oplus U_{2}$, where $U_{1}$ is finite dimensional. Suppose that $J \in C^{1}(U, \mathbb{R})$ satisfies the Palais-Smale condition and the following:

$\left(J_{1}\right)$ There are positive constants $c$ and $\rho$ such that $\left.J\right|_{\partial B_{\rho}(0) \cap U_{2}} \geq c$.

$\left(J_{2}\right)$ There are $\mu \in \partial B_{1}(0) \cap U_{2}$ and a positive constant $\hat{c} \geq \rho$ such that $\left.J\right|_{\partial \Omega} \leq 0$, where $\Omega=\left(\bar{B}_{\hat{c}}(0) \cap U_{1}\right) \oplus\{s \mu \mid 0<s<\hat{c}\}$.

Then $J$ possesses a critical value $c_{0} \geq c$, where

$$
c_{0}=\inf _{d \in \Upsilon} \sup _{u \in \Omega} J(d(u))
$$

and $\Upsilon=\left\{d \in C(\bar{\Omega}, U)|d|_{\partial \Omega}=i d\right\}$, where id denotes the identity operator.

Lemma 3.2 (Clark theorem [21]) Let $U$ be a real Banach space, $J \in C^{1}(U, \mathbb{R})$, with $J$ being even, bounded from below and satisfying the Palais-Smale condition. Assume $J(0)=0$, there is a set $\Gamma \subset U$ such that $\Gamma$ is homeomorphic to $S^{k-1}(k-1$ dimension unit sphere) by an odd map, and $\sup _{\Gamma} J<0$. Then $J$ has at least $k$ distinct pairs of nonzero critical points.

Lemma 3.3 Assume that $(r)$ and $\left(F_{1}\right)-\left(F_{3}\right)$ are satisfied. Then the functional $J$ satisfies the Palais-Smale condition.

Proof Let $\left\{u^{(j)}\right\}_{j \in \mathbb{N}} \subset U$ be such that $\left\{J\left(u^{(j)}\right)\right\}_{j \in \mathbb{N}}$ is bounded and $J^{\prime}\left(u^{(j)}\right) \rightarrow 0$ as $j \rightarrow \infty$. Then there is a constant $A>0$ such that

$$
-A \leq J\left(u^{(j)}\right) \leq A, \quad \forall j \in \mathbb{N}
$$

From $\left(F_{2}\right)$ and (2.3), for any $\left\{u^{(j)}\right\}_{j \in \mathbb{N}} \subset U$, we have

$$
\begin{aligned}
-A & \leq J\left(u^{(j)}\right)=\frac{1}{2}\left(u^{(j)}\right)^{*} M u^{(j)}-\sum_{n=1}^{k} F\left(n, u^{(j)}\right) \\
& \leq \frac{\lambda \max }{2}\left\|u^{(j)}\right\|^{2}-\sum_{n=1}^{k}\left[a_{2}\left(u^{(j)}\right)^{2}-a_{3}\right] \\
& =\left(\frac{\lambda_{\max }}{2}-a_{2}\right)\left\|u^{(j)}\right\|^{2}+a_{3} k .
\end{aligned}
$$

Then

$$
\left(a_{2}-\frac{\lambda_{\max }}{2}\right)\left\|u^{(j)}\right\|^{2} \leq A+a_{3} k .
$$


It comes from $a_{2} \in\left(\frac{\lambda_{\max }}{2},+\infty\right)$ that we can find a constant $B>0$ such that, for any $j \in \mathbb{N}$, $\left\|u^{(j)}\right\| \leq B$. Thus, we know that the sequence $\left\{u^{(j)}\right\}_{j \in \mathbb{N}}$ is bounded in the $k$ dimensional space $U$. Therefore, the Palais-Smale condition holds.

\section{Proofs of theorems}

Proof of Theorem 1.1 Obviously, $F(n, 0)=0$ and $f(n, 0)=0$ for any $n \in \mathbb{Z}[1, k]$ via $\left(F_{1}\right)$ and $\left(F_{2}\right)$. Hence, $u=0$ is a trivial solution of BVP (1.1) with (1.2).

It comes from Lemma 3.3 that $J(u)$ is bounded from above in $U$. Let

$$
\bar{J}=\sup _{u \in U} J(u)
$$

Therefore, there exists a sequence $\left\{u^{(j)}\right\}$ on $U$ such that

$$
\bar{J}=\lim _{j \rightarrow \infty} J\left(u^{(j)}\right)
$$

What is more, from the proof of Lemma 3.3, we have

$$
J(u) \leq\left(\frac{\lambda_{\max }}{2}-a_{2}\right)\|u\|^{2}+a_{3} k, \quad \forall u \in U .
$$

This implies that $\lim _{\|u\| \rightarrow+\infty} J(u)=-\infty$. Thus, $\left\{u^{(j)}\right\}$ is bounded. Then $\left\{u^{(j)}\right\}$ has a convergent subsequence defined by $\left\{u^{\left(j_{n}\right)}\right\}$. Set

$$
\bar{u}=\lim _{n \rightarrow+\infty} u^{\left(j_{n}\right)}
$$

Due to the continuity of $J(u)$ in $u$, there must be a point $\bar{u} \in U, J(\bar{u})=\bar{J}$. Clearly, $\bar{u} \in U$ is a critical point of $J(u)$.

From $\left(F_{1}\right)$, for any $u \in Q,\|u\| \leq \delta_{1}$, we have

$$
\begin{aligned}
J(u) & =\frac{1}{2} u^{*} M u-\sum_{n=1}^{k} F\left(n, u_{n}\right) \\
& \geq \frac{\lambda_{\min }}{2}\|u\|^{2}-a_{1} \sum_{n=1}^{k} u_{n}^{2} \\
& \geq\left(\frac{\lambda_{\min }}{2}-a_{1}\right)\|u\|^{2} .
\end{aligned}
$$

Denote

$$
c=\left(\frac{\lambda_{\min }}{2}-a_{1}\right) \delta_{1}^{2} .
$$

We have

$$
J(u) \geq c, \quad \forall u \in Q \cap \partial B_{\delta_{1}}(0) .
$$


Thus, there are constants $c>0$ and $\delta_{1}>0$ such that $\left.J\right|_{\partial B_{\delta_{1}}(0) \cap Q} \geq c$. Assumption $\left(J_{1}\right)$ of the linking theorem is satisfied.

In view of $M u=0$, for all $u \in P$, we have

$$
J(u)=\frac{1}{2} u^{*} M u-\sum_{n=1}^{k} F\left(n, u_{n}\right)=-\sum_{n=1}^{k} F\left(n, u_{n}\right) \leq 0 .
$$

Hence, $\bar{u} \notin P$ and the critical point $\bar{u}$ of $J(u)$ corresponding to the critical value $\bar{J}$ is a nontrivial solution of BVP (1.1) with (1.2).

In the light of Lemmas 3.1 and 3.3, it is sufficient to verify condition $\left(J_{2}\right)$.

Choose $\alpha \in \partial B_{1}(0) \cap Q$, for any $\beta \in P$ and $s \in \mathbb{R}$, let $u=s \alpha+\beta$. By $\left(F_{2}\right)$, we have

$$
\begin{aligned}
J(u) & =\frac{1}{2}(s \alpha+\beta)^{*} M(s \alpha+\beta)-\sum_{n=1}^{k} F\left(n, s \alpha_{n}+\beta_{n}\right) \\
& \leq \frac{1}{2}(s \alpha)^{*} M(s \alpha)-\sum_{n=1}^{k}\left[a_{2}\left(s \alpha_{n}+\beta_{n}\right)^{2}-a_{3}\right] \\
& \leq \frac{\lambda_{\max } s^{2}}{2}-a_{2} \sum_{n=1}^{k}\left(s \alpha_{n}+\beta_{n}\right)^{2}+a_{3} k \\
& =\left(\frac{\lambda_{\max }}{2}-a_{2}\right) s^{2}-a_{2}\|\beta\|^{2}+a_{3} k \\
& \leq-a_{2}\|\beta\|^{2}+a_{3} k .
\end{aligned}
$$

Consequently, there is some positive constant $\chi>\delta_{1}$ such that

$$
J(u) \leq 0, \quad \forall u \in \partial \Omega,
$$

where $\Omega=\left(\bar{B}_{\chi}(0) \cap Q\right) \oplus\{s \alpha \mid 0<s<\chi\}$. Applying the linking theorem, $J(u)$ has a critical value $c_{0} \geq c>0$, where

$$
c_{0}=\inf _{d \in \Upsilon} \sup _{u \in \Omega} J(d(u))
$$

and $\Upsilon=\left\{d \in C(\bar{\Omega}, U)|d|_{\partial \Omega}=i d\right\}$.

Similar to the proof of Theorem 1.1 in [4], we can prove that BVP (1.1) with (1.2) admits at least three solutions, and so we omit it.

Remark 4.1 Note that $\left(F_{3}\right)$ implies $\left(F_{1}\right)$. Similar to the above argument, we can also prove Theorem 1.2. For simplicity, we omit its proof.

Proof of Theorem 1.3 Obviously $J \in C^{1}(U, \mathbb{R}), J$ is even, and $J(0)=0$. From Lemma 3.3, $J$ satisfies the Palais-Smale condition. By the proof of Theorem 1.1, we have that $J$ is bounded from below. On account of Lemma 3.2, it is sufficient to find a set $\Gamma$ and an odd map such that $\Gamma$ is homeomorphic to $S^{q-1}$ by an odd map.

Choose

$$
\Gamma=\partial B_{\delta_{1}}(0) \cap Q
$$


Clearly, $\Gamma$ is homeomorphic to $S^{q-1}$ by an odd map. It is comes from (4.2) that $\sup _{\Gamma}(-J)<0$. On the basis of Lemma 3.2, $J$ has at least $q$ distinct pairs of nonzero critical points. For this reason, BVP (1.1) with (1.2) admits at least $q$ distinct pairs of nontrivial solutions. The proof of Theorem 1.3 is complete.

Remark 4.2 By virtue of Theorem 1.1, the conclusion of Theorem 1.4 is clearly right. As a result of Corollary Theorem 1.3, the results of Corollary 1.1 are evidently correct.

\section{Examples}

Firstly, our example illustrates Theorem 1.1.

Example 5.1 Consider the equation

$$
\Delta^{4} u_{n-2}-\Delta^{2} u_{n-1}=u_{n}^{6}, \quad n \in \mathbb{Z}[1,3]
$$

with boundary value conditions

$$
u_{-1}=u_{2}, \quad \Delta u_{-1}=\Delta u_{2}, \quad \Delta^{2} u_{-1}=\Delta^{2} u_{2}, \quad \Delta^{3} u_{-1}=\Delta^{3} u_{2} .
$$

We have

$$
r_{n} \equiv 1, \quad f\left(n, u_{n}\right)=u_{n}^{6},
$$

and

$$
F\left(n, u_{n}\right)=\frac{1}{7} u_{n}^{7}
$$

Also,

$$
M=S+R=\left(\begin{array}{ccc}
8 & -4 & -4 \\
-4 & 8 & -4 \\
-4 & -4 & 8
\end{array}\right)
$$

and the eigenvalues of $M$ are $\lambda_{1}=0, \lambda_{2}=12$, and $\lambda_{3}=12$. It is easy to verify that all the conditions of Theorem 1.1 are satisfied and then BVP (5.1) with (5.2) admits at least three solutions.

As an example of Theorem 1.3, we have the following.

Example 5.2 Consider the equation

$$
\Delta^{4} u_{n-2}-2 \Delta^{2} u_{n-1}=10 u_{n}^{9}, \quad n \in \mathbb{Z}[1,4]
$$

with boundary value conditions

$$
u_{-1}=u_{3}, \quad \Delta u_{-1}=\Delta u_{3}, \quad \Delta^{2} u_{-1}=\Delta^{2} u_{3}, \quad \Delta^{3} u_{-1}=\Delta^{3} u_{3} .
$$


We have

$$
r_{n} \equiv 2, \quad f\left(n, u_{n}\right)=10 u_{n}^{9}
$$

and

$$
F\left(n, u_{n}\right)=u_{n}^{10}
$$

Also,

$$
M=S+R=\left(\begin{array}{cccc}
10 & -6 & 2 & -6 \\
-6 & 10 & -6 & 2 \\
2 & -6 & 10 & -6 \\
-6 & 2 & -6 & 10
\end{array}\right)
$$

and the eigenvalues of $M$ are $\lambda_{1}=0, \lambda_{2}=8, \lambda_{3}=8$, and $\lambda_{4}=24$. It is easy to verify that all the conditions of Theorem 1.3 are satisfied, and then BVP (5.3) with (5.4) admits at least three distinct pairs of nontrivial solutions.

\section{Acknowledgements}

The authors are extremely grateful to the referees and the editors for their careful reading and making some valuable comments and suggestions on the manuscript. This work was carried out while visiting Central South University. The author Haiping Shi wishes to thank Professor Xianhua Tang for his invitation.

\section{Funding}

This project is supported by the National Natural Science Foundation of China (No. 11501194).

\section{Competing interests}

The authors declare that there is no conflict of interests regarding the publication of this paper

\section{Authors' contributions}

All authors contributed equally and significantly in writing this article. All authors read and approved the final manuscript.

\section{Author details}

'College of Continuing Education and Open College, Guangdong University of Foreign Studies, Guangzhou, China. ${ }^{2}$ Science College, Hunan Agricultural University, Changsha, China. ${ }^{3}$ School of Economics and Managment, South China Normal University, Guangzhou, China. ${ }^{4}$ Modern Business and Management Department, Guangdong Construction Polytechnic, Guangzhou, China. ${ }^{5}$ School of Mathematics and Statistics, Central South University, Changsha, China.

\section{Publisher's Note}

Springer Nature remains neutral with regard to jurisdictional claims in published maps and institutional affiliations.

Received: 15 June 2018 Accepted: 8 November 2018 Published online: 20 November 2018

\section{References}

1. Agarwal, P., Al-Omari, S.K.Q., Choi, J.: Real covering of the generalized Hankel-Clifford transform of Fox kernel type of a class of Boehmians. Bull. Korean Math. Soc. 52(5), 1607-1619 (2015)

2. Agarwal, P., Al-Omari, S.K.Q., Park, P.: An extension of some variant of Meijer type integrals in the class of Boehmians. J. Inequal. Appl. 2016, 70 (2016)

3. Agarwal, R.P.: Difference Equations and Inequalities: Theory, Methods and Applications. Dekker, New York (2000)

4. Chen, P., He, X.F.: Existence and multiplicity of homoclinic solutions for second-order nonlinear difference equations with Jacobi operators. Math. Methods Appl. Sci. 39(18), 5705-5719 (2016)

5. Chen, P., Tang, X.H.: Existence of infinitely many homoclinic orbits for fourth-order difference systems containing both advance and retardation. Appl. Math. Comput. 217(9), 4408-4415 (2011)

6. Chen, P., Tang, X.H.: Existence and multiplicity of homoclinic orbits for $2 n$ th-order nonlinear difference equations containing both many advances and retardations. J. Math. Anal. Appl. 381 (2), 485-505 (2011)

7. Chen, P., Tang, X.H.: Existence of homoclinic solutions for some second-order discrete Hamiltonian systems. J. Differ. Equ. Appl. 19(4), 633-648 (2013)

8. Došlá, Z., Krejčová, J., Marini, M.: Minimal and maximal solutions of fourth-order nonlinear difference equations. J. Differ. Equ. Appl. 21(3), 255-268 (2015) 
9. Graef, J.H., Kong, L.J., Wang, M.: Multiple solutions to a periodic boundary value problem for a nonlinear discrete fourth order equation. Adv. Dyn. Syst. Appl. 8(2), 203-215 (2013)

10. Graef, J.H., Kong, L.J., Wang, M.: Two nontrivial solutions for a discrete fourth order periodic boundary value problem. Commun. Appl. Anal. 19, 487-496 (2015)

11. Guo, C.J., Guo, C.X., Ahmed, S., Liu, X.F.: Moment stability for nonlinear stochastic growth kinetics of breast cancer stem cells with time-delays. Dyn. Contin. Discrete Impuls. Syst., Ser. B, Appl. Algorithms 21(8), 2473-2489 (2016)

12. Guo, C.J., O'Regan, D., Wang, C.J., Agarwal, R.P.: Existence of homoclinic orbits of superquadratic second-order Hamiltonian systems. Z. Anal. Anwend. 34(1), 27-41 (2015)

13. Guo, C.J., O'Regan, D., Xu, Y.T., Agarwal, R.P.: Homoclinic orbits for a singular second-order neutral differential equation. J. Math. Anal. Appl. 366(2), 550-560 (2010)

14. Guo, C.J., Xu, Y.T.: Existence of periodic solutions for a class of second order differential equation with deviating argument. J. Appl. Math. Comput. 28(1-2), 425-433 (2008)

15. He, T.S., Chen, C.Y., Huang, Y.H., Hou, C.H.: Infinitely many sign-changing solutions for $p$-Laplacian Neumann problems with indefinite weight. Appl. Math. Lett. 39, 73-79 (2015)

16. He, T.S., Su, Y.L.: On discrete fourth-order boundary value problems with three parameters. J. Comput. Appl. Math. 233(10), 2506-2520 (2010)

17. He, T.S., Zhou, Y.W., Xu, Y.T., Chen, C.Y.: Sign-changing solutions for discrete second-order periodic boundary value problems. Bull. Malays. Math. Sci. Soc. 38(1), 181-195 (2015)

18. Lin, G.H., Zhou, Z:: Homoclinic solutions of discrete $\phi$-Laplacian equations with mixed nonlinearities. Commun. Pure Appl. Anal. 17(5), 1723-1747 (2018)

19. Liu, X., Zhang, Y.B., Shi, H.P.: Existence and nonexistence results for a fourth-order discrete Dirichlet boundary value problem. Hacet. J. Math. Stat. 44(4), 855-866 (2015)

20. Long, Y.H., Chen, J.L.: Existence of multiple solutions to second-order discrete Neumann boundary value problems. Appl. Math. Lett. 83, 7-14 (2018)

21. Mawhin, J., Willem, M.: Critical Point Theory and Hamiltonian Systems. Springer, New York (1989)

22. Peterson, A., Ridenhour, J.: The (2, 2)-disconjugacy of a fourth order difference equation. J. Differ. Equ. Appl. 1(1), 87-93 (1995)

23. Rabinowitz, P.H.: Minimax Methods in Critical Point Theory with Applications to Differential Equations. Am. Math. Soc., Providence (1986)

24. Tang, X.H., Chen, S.T.: Ground state solutions of Nehari-Pohozaev type for Schrödinger-Poisson problems with general potentials. Discrete Contin. Dyn. Syst. 37(9), 4973-5002 (2017)

25. Tang, X.H., Chen, S.T.: Ground state solutions of Nehari-Pohozaev type for Kirchhoff-type problems with general potentials. Calc. Var. Partial Differ. Equ. 56(4), 110-134 (2017)

26. Xia, F.: Homoclinic solutions for second-order nonlinear difference equations with Jacobi operators. Electron. J. Differ. Equ. 2017, 94 (2017)

27. Xia, F.: Existence of periodic solutions for higher order difference equations containing both many advances and retardations. Rev. R. Acad. Cienc. Exactas Fís. Nat., Ser. A Mat. 112(1), 239-249 (2018)

28. Yang, L.W.: Existence of homoclinic orbits for fourth-order p-Laplacian difference equations. Indag. Math. 27(3), 879-892 (2016)

29. Yao, Q:: Positive solutions of a nonlinear elastic beam equation rigidly fastened on the left and simply supported on the right. Nonlinear Anal. 69(5-6), 1570-1580 (2008)

30. Zhou, Z., Ma, D.F.: Multiplicity results of breathers for the discrete nonlinear Schrödinger equations with unbounded potentials. Sci. China Math. 58(4), 781-790 (2015)

31. Zhou, Z., Yu, J.S., Chen, Y.M.: Homoclinic solutions in periodic difference equations with saturable nonlinearity. Sci. China Math. 54(1), 83-93 (2011)

\section{Submit your manuscript to a SpringerOpen ${ }^{\circ}$ journal and benefit from:}

- Convenient online submission

- Rigorous peer review

- Open access: articles freely available online

- High visibility within the field

Retaining the copyright to your article

Submit your next manuscript at $\gg$ springeropen.com 